Corresponding Author: Deepti Arora;

email: deepti.a15@gmail.com

Received 02 July 2021

Accepted 14 September 2021

Published 30 September 2021

Production and Hosting by Knowledge E

(c) Ina Garg et al.. This article is distributed under the terms of the Creative Commons

Attribution License, which permits unrestricted use and redistribution provided that the original author and source are credited.

Editor-in-Chief:

Prof. Mohammad A. M. Ibnouf

\section{Association of Serum Adenosine Deaminase Levels in Cytologically Suggested Cases of Tubercular Lymphadenitis: The Experience of a Tertiary Care Centre}

Ina Garg, Deepti Arora, Himanshu Joshi, Ashutosh Kumar, and Seema Awasthi

Department of Pathology, Teerthanker Mahaveer Medical College \& Research Centre (TMMC\&RC), Moradabad, Uttar Pradesh, India

ORCID:

Deepti Arora: $h$ ttp://orcid.org/0000-0001-5461-8592

\section{Abstract}

Background: Tuberculosis (TB), a communicable disease, caused by Mycobacterium tuberculosis requires a simple, rapid test, which can be easily carried out in a laboratory. Unfortunately, despite a battery of investigations, no definite test is available till date. Adenosine deaminase (ADA), a biochemical marker has been proposed as a useful surrogate marker for TB as its levels can be measured in body fluids.

Methods: A one-and-a-half-year prospective study of 154 cases presenting with lymphadenitis from January 2019 to June 2020 was undertaken. Using cytology, lymphadenitis subjects were divided into two groups: Tubercular (104 patients) as a case group and Reactive (50 patients) as a control group. All cases were followed by serum ADA assay by colorimetric method. Nonparametric tests were performed to compare the two groups.

Results: The mean age of the participants was $28.99 \pm 13.26$ years with a F:M ratio of 1.81:1. Involvement of cervical lymph nodes was most frequent (89.42\% cases). The mean S.ADA level for tubercular and reactive lymphadenitis was $41.71 \pm 11.53 \mathrm{U} / \mathrm{L}$ and $21.16 \pm 4.16 \mathrm{U} / \mathrm{L}$, respectively $(P$-value $<0.05)$. The cut-off value calculated was 32.6 U/L. The sensitivity, specificity, PPV, NPV, and accuracy were calculated as $79.81 \%$, $100 \%, 100 \%, 70.42 \%$, and $86.36 \%$, respectively.

Conclusion: A statistically significant increase was found in serum ADA levels in tubercular lymphadenitis cases compared to reactive lymphadenitis. Hence, it can be used as an adjunct to FNAC and is a fairly sensitive and specific test. Since it is difficult to always demonstrate AFB in FNAC smears, ADA can be helpful in establishing a definite diagnosis despite smear negativity.

Keywords: adenosine deaminase, lymphadenitis, tuberculosis

\section{Introduction}

Tuberculosis (TB), a transmittable disease, is amongst the top 10 worldwide causes of S OPEN ACCESS 
over HIV/ AIDS [1]. In 1993, TB was declared as a public health emergency on a global level by WHO [2]. Depending upon the locus of involvement, TB can have two different manifestations clinically: Pulmonary TB, wherein infection is situated in the lungs and Extrapulmonary TB, involving any site other than the lungs, for example, abdomen, lymph nodes, meninges, skin, or genito-urinary tract [3]. The most common manifestation of mycobacterial extra-pulmonary disease is the involvement of peripheral lymph nodes, usually of the cervical area [4]. Several research studies conducted in India have also shown Mycobacterium tuberculosis (M. TB) to be the pathogen that was commonly isolated from mycobacterial lymphadenitis taking in account most of the cases [5-7].

An expeditious diagnosis is a mainstay for an efficient TB curbing program. A variety of methods for pulmonary TB detection are available but these do not yield sufficient specificity or sensitivity. The sensitivities of TB diagnosis using culture and ZiehlNeelsen staining are $8-49 \%$ and $10-40 \%$, respectively [8]. The gold standard test for the detection of TB is the culture of the causative organism [9]. The technique of choice for extrapulmonary TB detection is histopathological examination. This includes excisional biopsy followed by culture and Ziehl-Neelsen stain. However, being an invasive modality and due to the lack of availability in peripheral centers makes its feasibility limited in low-resource areas [10].

Fine-needle aspiration is an economic, rapid, and simple technique that has high specificity and sensitivity for extrapulmonary TB and can be performed on an outpatient basis. When extrapulmonary TB is considered, Acid Fast Bacilli (AFB) positivity is not always found due to paucibacillary nature particularly for those cases which are associated with immunosuppression. In endemic nations like India, even without AFB positivity, the mere presence of granuloma with/without caseation and with/without giant cells-Langhan's type is taken as and treated for TB [11].

We need an easy, reliable, and rapid test that can easily be executed in the laboratory setting. Therefore, we thought of using serum Adenosine deaminase (ADA) as an adjunctive diagnostic tool considering the fact that fluid ADA levels are already in use for tubercular effusions since 1978. Various articles have found the test to have high sensitivity-92\% and specificity-89\% for early detection of extrapulmonary TB in cases of tuberculous pericarditis, meningitis, pleuritis, and ascites [12]. Although a good amount of data has validated the yield of ADA in tubercular effusions, we cannot always access these fluids. Instead, serum levels can be easily performed but there is a paucity of literature correlating it to tubercular lymphadenitis. Therefore, we undertook this 
study intending to demonstrate the association of serum ADA activity in cytologically suggested tubercular lymphadenitis cases.

\section{Material and Methods}

This prospective cross-sectional study was carried out in the Department of Pathology of a tertiary care center from January 2019 to June 2020. All peripheral lymph node fine needle aspirations with clinical suspicion of TB during this period were included in this study. A total of 154 subjects were enrolled.

\subsection{Inclusion criteria}

1. Both genders and all age groups were included.

2. Patients referred for fine needle aspiration cytology of lymph nodes with clinical and cytological suspicion of tubercular lymphadenitis or reactive lymphadenitis.

\subsection{Exclusion Criteria}

1. All causes of raised serum ADA levels other than TB.

2. Patients who did not give consent for the study.

The study was approved by the Ethical and Research Committee of the Institute. Patients were selected in accordance with predefined inclusion and exclusion criteria of the study. All selected patients were briefed on the nature of the study and a written informed consent for FNAC and serum ADA estimation was obtained. The relevant clinical details, demographic data of the patients such as the name, age, and sex were recorded.

The prepared smears were stained by Giemsa and Ziehl-Neelsen stain. All the stained slides were thoroughly analyzed by two independent trained pathologists with more than eight years' experience and were examined for the features of tubercular and reactive lymphadenitis. Out of the 154 subjects, 104 with cytologically suggested diagnosis of tubercular lymphadenitis were taken as the case group while 50 age- and sex-matched individuals presenting as reactive lymphadenitis were included as control group.

The tubercular lymphadenitis cases were segregated based on the cytologic patterns described by Dasgupta et al. [13] as follows: 
1. Pattern A: Epithelioid granuloma without necrosis,

2. Pattern B: Epithelioid granuloma with necrosis, and

3. Pattern C: Necrosis with/without neutrophilic infiltrate.

A 2-ml blood sample from the patient was then collected into a plain vial and serum ADA estimation using non-Guisti's method was done (Adenosine deaminase Assay kit, ERBA Mannheim, TransAsia Bio-medicals Ltd.) on semi-automated analyzer. Cytologically suggested cases of TB lymphadenitis were followed-up to assess clinical response to therapy (no evening rise of temperature, improvement in appetite, and weight gain) after one month, and in case of no response, the case was excluded.

\subsection{Statistical analysis}

The data obtained were analyzed using descriptive statistics, unpaired $t$-test, oneway ANOVA test, and Chi-square test. SPSS program for Windows, version 25.0 was employed for statistical analysis. $P$-value $<0.05$ was considered as statistically significant for all statistical tests. To determine optimal cut-off value of serum ADA levels in tubercular lymphadenitis, a receiver operating characteristics (ROC) analysis was done and area under the curve and its standard deviation (AUC_SD), sensitivity, specificity, positive predictive value (PPV), negative predictive value (NPV), and accuracy were calculated.

\section{Results}

Out of the 104 patients that presented with cytological features suggestive of tubercular lymphadenitis, 37 were male and 67 female. Female dominance was present in our study with a F:M ratio of 1.81:1. The age of the patients ranged from 5 to 70 years. The mean age in the case group was $28.99 \pm 13.26$ years.

The involvement of cervical lymph nodes was most frequent and was observed in $89.42 \%$ of cases. On FNAC, pattern B (Figure 1) (69 cases, 66\%) was most frequently encountered, followed by pattern A (Figure 2 ) ( 25 cases, $25 \%$ ) and then by pattern $C$ (Figure 3) (9 cases, 9\%). Out of the 104 cases of tubercular lymphadenitis, AFB positivity (Figure 4 ) was seen in $24.04 \%$ of the cases. Maximum AFB positivity was observed in cases belonging to pattern $C$.

The mean serum ADA level in tubercular lymphadenitis was $41.71 \pm 11.53 \mathrm{U} / \mathrm{L}$ and in reactive lymphadenitis was $21.16 \pm 4.16 \mathrm{U} / \mathrm{L}$ (Figure 5). 
The mean serum ADA level was statistically more significant among tubercular lymphadenitis compared to reactive lymphadenitis ( $p$-value $<0.001$ ) (Table 1$)$. No significant difference in the distribution of serum ADA levels between AFB-positive and -negative groups was observed $(p$-value $=0.669)($ Table 2$)$. No significant difference was reported in the mean ADA levels among patterns $A, B$, and $C(p$-value $=0.438)$ (Table 3$)$.

The optimal cut-off value for serum ADA level was calculated to differentiate cases having tubercular lymphadenitis from reactive lymphadenitis using ROC curve. The optimal cut-off value for identifying tubercular lymphadenitis was found to be $32.6 \mathrm{U} / \mathrm{L}$ with resulting sensitivity (79.81\%,), specificity (100\%), PPV (100\%), NPV (70.42\%), and accuracy for serum ADA level calculated as $86.36 \%$ (Figure 6).

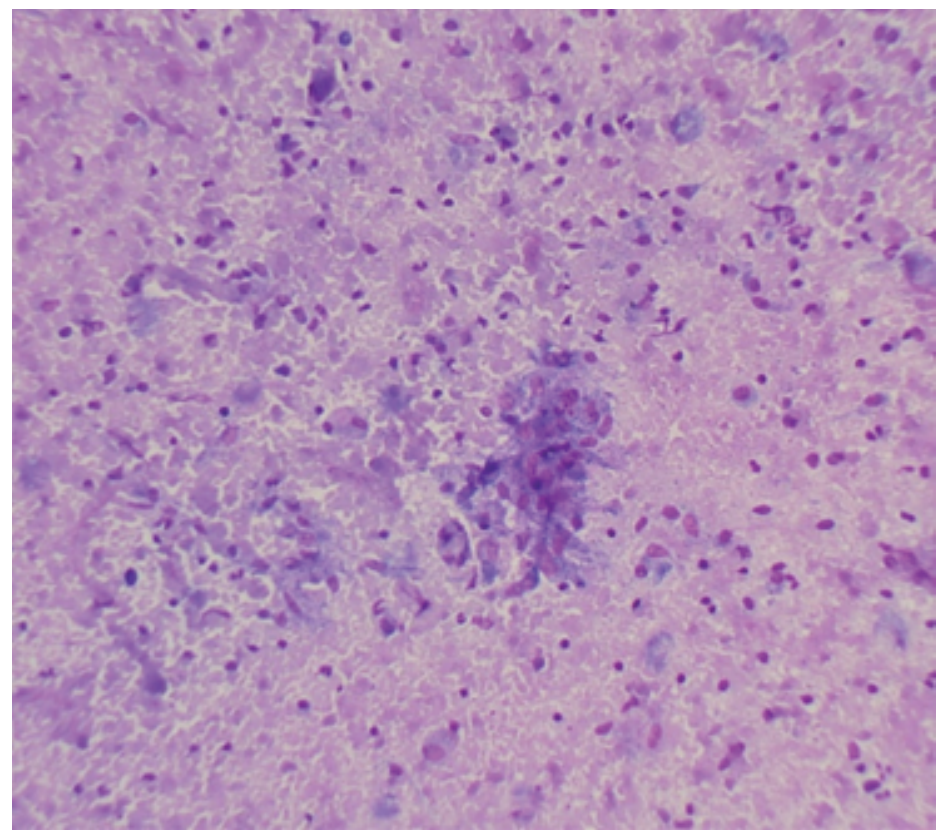

Figure 1: Photomicrograph showing Epithelioid cell granuloma with a necrotic background (Pattern B); 100x (MGG).

TABLE 1: Comparison of mean serum ADA levels in reactive lymphadenitis (control) and tubercular lymphadenitis (cases) (Department of Pathology, TMU from January 2019 to June 2020) $n=154$.

\begin{tabular}{|l|l|l|l|l|l|} 
& Mean (U/L) & $\begin{array}{l}\text { Standard } \\
\text { deviation }\end{array}$ & $\begin{array}{l}\text { Mean } \\
\text { Difference }\end{array}$ & t-test value & $P$-value \\
\hline Reactive Lymphadenitis & 21.16 & 4.16 & -20.55 & -12.213 & $<0.001^{*}$ \\
\hline Tubercular Lymphadenitis & 41.71 & 11.53 & & & \\
\hline
\end{tabular}

Unpaired $t$-test; *Significant difference. 


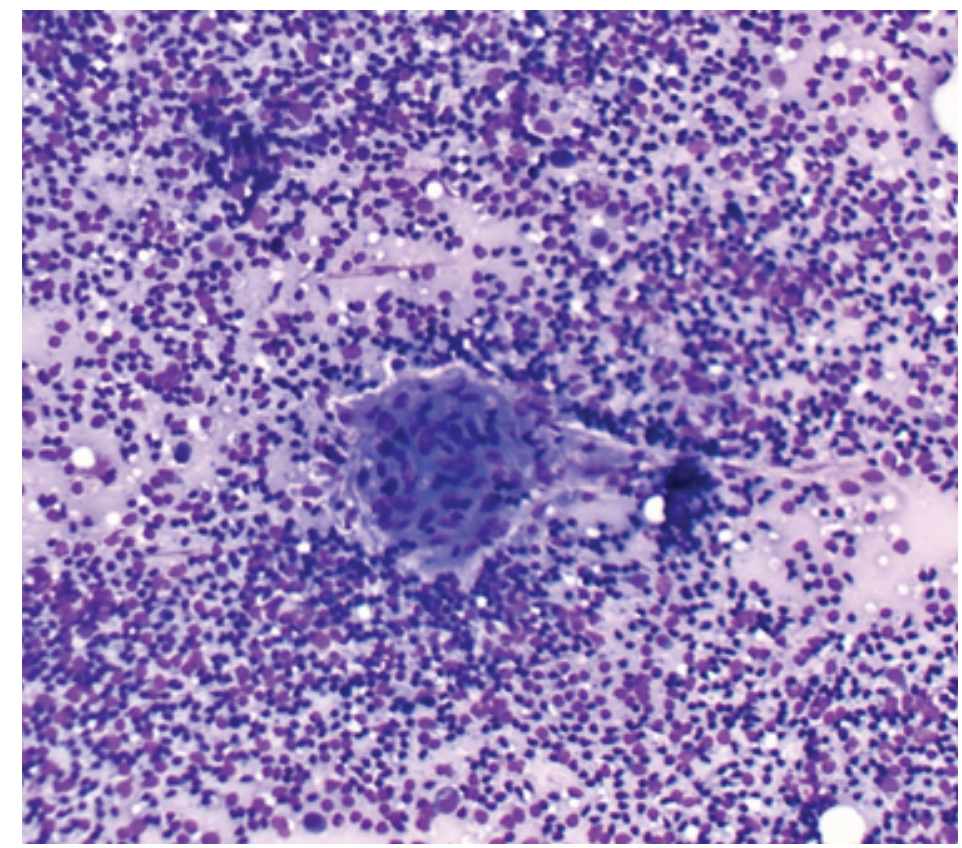

Figure 2: Photomicrograph showing epithelioid cell granuloma (Pattern A); 100x (MGG).

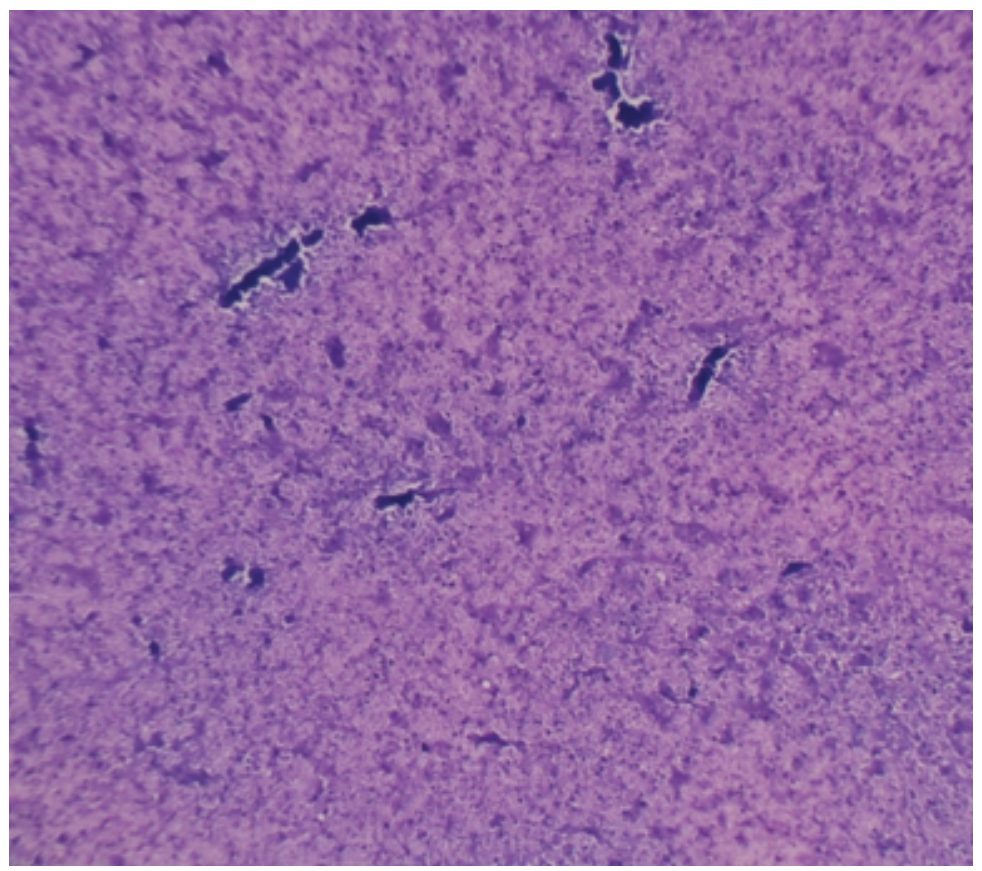

Figure 3: Photomicrograph showing caseous necrosis (Pattern C); 100x (MGG).

\section{Discussion}

TB has been a public health emergency as well as the chief cause of fatality for a long time. The mainstay for controlling this infection is an early unerring diagnosis followed by an appropriate prompt treatment [1]. In the case of tubercular lymphadenitis, a proper diagnosis will require either an FNA-obtained material or a biopsy which will 


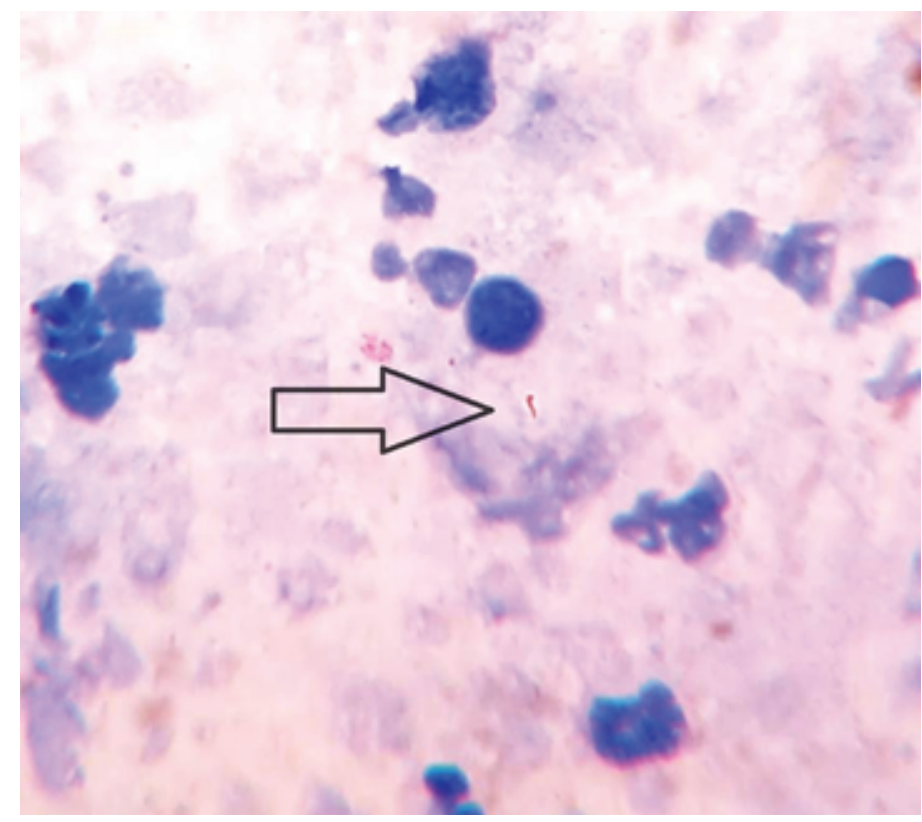

Figure 4: Photomicrograph showing acid fast bacilli; oil-immersion 1000x (Ziehl-Neelsen stain).

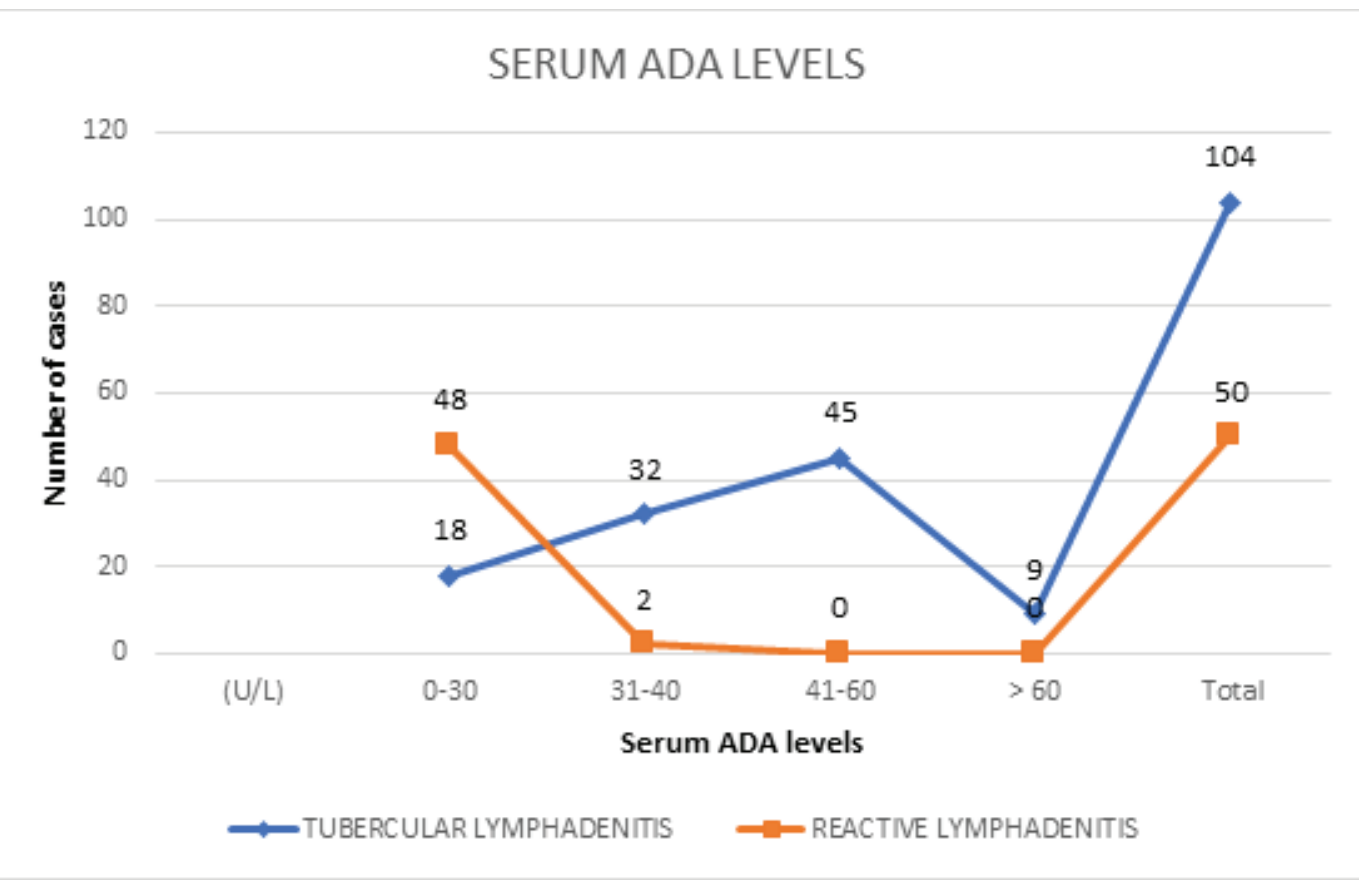

Figure 5: Comparison of serum ADA levels between cases of tubercular and reactive lymphadenitis (Department of Pathology, TMU, January 2019 to June 2020) $n=104$

then be followed by culture, AFB confirmation via staining or other molecular techniques. Problem rises due to the paucity of demonstrable acid-fast bacilli at such locations thereby impeding a definite diagnosis. In such situations, we require an adjunctive tool that might facilitate a diagnosis $[9,10]$.

Serum ADA estimation is one such modality which, in addition to being noninvasive, is a simple method for disease detection. ADA can serve as an indicator of cell-mediated 


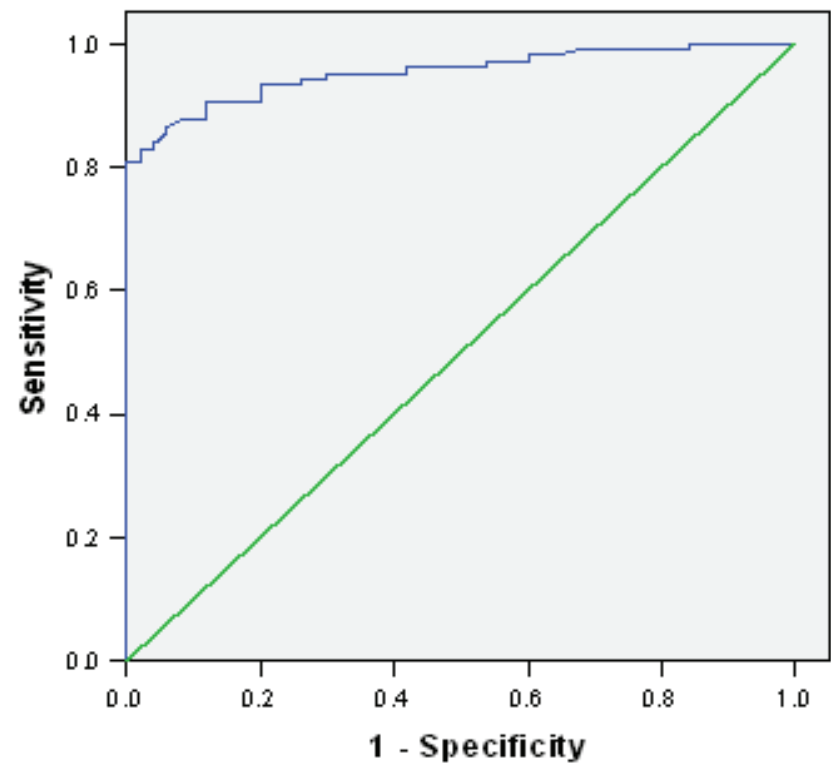

Figure 6: ROC analysis of optimal cut-off value for serum ADA level to differentiate cases of tubercular lymphadenitis from reactive lymphadenitis (Department of Pathology, TMU from January 2019 to June 2020) $n=104$.

The test result variable(s): ADA has at least one tie between the positive actual state group and the negative actual state group. Statistics may be biased

1. under the nonparametric assumption and

2. null hypothesis: true area $=0.5$.

AUC 0.953 (95\% Cl, 0.923-0.983, $\left.p<0.001^{*}\right)$, Sensitivity 79.81\% (70.81-87.04\%), Specificity 100\% (92.89$100.00 \%$ ), Positive Predictive Value $100 \%$, Accuracy $86.36 \%$ (79.91-91.36\%), and Negative Predictive Value $70.42 \%(61.90-77.72 \%)$.

TABLE 2: Correlation of serum ADA levels with AFB status (demonstration on Ziehl-Neelsen-stained smear) in cases of tubercular lymphadenitis (Department of Pathology, TMU from January 2019 to June 2020) $n=$ 154.

\begin{tabular}{l|l|l|l|l|l|}
\hline & \multicolumn{5}{c}{ SERUM ADA } \\
AFB & Mean (U/L) & $\begin{array}{l}\text { Std. } \\
\text { deviation }\end{array}$ & $\begin{array}{l}\text { Mean } \\
\text { difference }\end{array}$ & t-test value & $P$-value \\
\hline Positive & 40.95 & 10.41 & -1.02 & -0.388 & $0.699^{\#}$ \\
Negative & 41.97 & 11.93 & & & \\
\hline
\end{tabular}

Unpaired t-test; "Non-significant difference.

TABLE 3: Correlation of serum ADA levels with different cytological patterns of tubercular lymphadenitis (Department of Pathology, TMU from January 2019 to June 2020) $n=104$.

\begin{tabular}{l|l|l|l|l} 
& \multicolumn{4}{c}{ SERUM ADA } \\
Pattern & Mean & Standard deviation & F-value & P-value \\
\hline A & 44.21 & 11.42 & 0.833 & $0.438^{\#}$ \\
\hline B & 40.98 & 11.75 & & \\
C & 40.12 & 10.07 & &
\end{tabular}

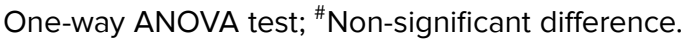

immunity. Its utility in pleural effusions was first demonstrated by Piras et al. [14]. Many studies [15-17] have established the utility of fluid ADA levels for the diagnosis of tuberculous peritoneal, pleural, pericardial effusions, and even in CSF but they are 
not always accessible. Therefore, we thought of using serum levels which we found elevated more in tubercular aetiologias as compared to nontubercular diseases.

In the present study, patients age ranged from 5 to 70 years. The age group most frequently involved among the patients with tubercular lymphadenitis was 21-30 years followed by 11-20 years. In this study, subjects belonging to the case group showed a slight female predominance with a female:male ratio of 1.81:1. Mugulkod et al., Khajuria et al., and purohit et al. [15-17] exhibited a minor female dominance as well.

Among the sites of involvement, the commonest was the cervical group of lymph nodes which comprised $89.42 \%$. In the study by Mugulkod et al. [15], of the 230 cases of extrapulmonary TB, 184 (80\%) were of lymphadenitis. Similar to our study, they also showed the cervical group of lymph nodes as most frequently involved.

The tubercular lymphadenitis cases were segregated based on the cytologic patterns described by Dasgupta et al. [13]. Of the 104 subjects, patterns A, B, and C were observed in 25,69 , and 9 cases, respectively. The commonest pattern in this study was pattern $B$ (66.34\% cases) showing epithelioid granuloma with necrosis. The same was observed by Bhattacharya et al. [18] and Khanna et al. [19] in 69.4\% and 50.5\% cases, respectively.

The presence of epithelioid cells was seen in $91.34 \%$ cases. Giant cells were noticed in $35.57 \%$ cases. In the present study, AFB positivity (Figure 4 ) was noted in $24.04 \%$ cases which was comparable to the ones reported as $25.65 \%$ and $26.1 \%$ by Mugulkod et al. [15] and Nassaji et al. [20], respectively.

The mean serum ADA levels in tubercular and reactive lymphadenitis were calculated as $41.71 \pm 11.53 \mathrm{U} / \mathrm{L}$ and $21.16 \pm 4.16 \mathrm{U} / \mathrm{L}$, respectively. Maximum cases, that is, $43.3 \%$ showed an ADA value in the range of $41-60 \mathrm{U} / \mathrm{L}$ followed by $30.8 \%$ cases in the range of 31-40 U/L. The mean serum ADA level was significantly high among tubercular lymphadenitis compared to reactive lymphadenitis.

Results similar to our study were also demonstrated in the studies by Sulakshana et al. [21], Ahmed et al. [22], Abdelsadek et al. [23], Mugulkod et al. [15], and Stevanovic et al. [3]. This was in contrast to the findings of Conde et al. [22]. They concluded that although serum ADA2 levels might help in differentiating pulmonary TB cases from controls, its utility in distinguishing it from other respiratory diseases is limited.

The cut-off value for tubercular lymphadenitis calculated using the ROC curve was $32.6 \mathrm{U} / \mathrm{L}$. At this cut-off, sensitivity, specificity, positive predictive value (PPV) and negative predictive value (NPV) were $79.81 \%, 100 \%, 100 \%$, and $70.42 \%$, respectively. In the present study, 20 (19.23\%) cases had a serum ADA level below the cut-off value of $32.6 \mathrm{U} / \mathrm{L}$ while none of the control group subjects crossed this value. In contrast, Salmanzadeh et al. [24] reported a sensitivity and specificity of 35\% and $91 \%$, 
respectively, for patients with pulmonary TB. They suggested that due to high specificity, ADA can be employed to rule out TB in suspected patients having a negative culture for AFB. Similarly, Farazi et al. [25] also reported a low sensitivity with a high PPV.

\section{Limitations}

The small sample size of the study may limit statistical relevance. Only patients with positive clinical response to treatment were included as cases while those who did not respond to treatment like MDR and XDR cases were excluded.

\section{Recommendations}

Further investigations with appropriate research methodology setup are needed to conclusively opine about the accuracy of serum ADA alone compared to ADA as an adjunct tool to FNAC in making TB diagnosis.

\section{Conclusion}

The raised serum ADA level can be considered as a reliable indicator of tubercular lymphadenitis and is a fairly sensitive and specific test. The use of non-Guisti's method makes it a rapid test that can be carried out easily even in remote areas with limited facilities. The best cut-off value calculated in our study was $32.6 \mathrm{U} / \mathrm{L}$ at which the sensitivity $(79.81 \%)$ and specificity $(100 \%)$ were found to be good enough for this test to help distinguish tubercular from non-tubercular aetiologias and also in detecting AFB smear-negative cases. So, in a country like India where TB is endemic, serum ADA can be used as an adjunct to FNAC for a definite diagnosis of tuberculous lymphadenitis especially in difficult situations or diagnostic dilemma. It can also be used as an early guide in subjecting the patient to FNAC considering the cut-off of $32.6 \mathrm{U} / \mathrm{L}$ below which we can say with the confidence of $86.36 \%$ that it is likely a non-tubercular or tubercular etiology.

\section{Acknowledgements}

The authors would like to thank all patients who kindly participated in the study. 


\section{Ethics Considerations}

Prior ethical approval was taken from the Institutional Ethics Committee (IEC), Teerthanker Mahaveer Medical College \& Research Centre (TMMC\&RC), Moradabad vide letter no. TMMC\&RC/IEC/18-19/020 dated 27/12/2018.

\section{Competing Interests}

The authors declare hereby that there are no conflicts of interest.

\section{Availability of Data and Material}

The raw data used during the current study are available from the corresponding author on reasonable request.

\section{Funding}

This research received no specific grant from any funding agency in the public, commercial, or not-for-profit sectors.

\section{References}

[1] World Health Organization (WHO). (2020). Global Tuberculosis Report 2020. Geneva: WHO. Retrieved from: https://www.who.int/tb/publications/global_report/ en/

[2] Grange, J. M. and Zumla, A. (2002). The global emergency of tuberculosis: what is the cause? Journal of the Royal Society for the Promotion of Health, vol. 122, no. 2.

[3] Stevanovic, G., Pelemis, M., Pavlovic, M., et al. (2011). Significance of adenosine deaminase serum concentration in the diagnosis of extra-pulmonary tuberculosis. Journal of IMAB, vol. 17, no. 2011, pp. 130-134.

[4] Golden, M. P. and Vikram, H. R. (2005). Extrapulmonary tuberculosis: an overview. American Family Physician, vol. 72, no. 9, pp. 1761-1768.

[5] Jawahar, M. S., Rajaram, K., Sivasubramanian, S., et al. (2005). Treatment of lymph node tuberculosis - a randomized clinical trial of two 6-month regimens. Tropical Medicine \& International Health, vol. 10, no. 11, pp. 1090-1098. 
[6] Subrahmanyam, M. (1993). Role of surgery and chemotherapy for peripheral lymph node tuberculosis. British Journal of Surgery, vol. 80, no. 12, pp. 1547-1548.

[7] Dandapat, M. C., Mishra, B. M., Dash, S. P., et al. (1990). Peripheral lymph node tuberculosis: a review of 80 cases. British Journal of Surgery, vol. 77, no. 8, pp. 911-912.

[8] Jay, S. J. (1985). Diagnostic procedures for pleural disease. Clinics in Chest Medicine, vol. 6 , no. 1 , pp. 33-48.

[9] Achkar, J. M., Lawn, S. D., Moosa, M.-Y. S., et al. (2011). Adjunctive tests for diagnosis of tuberculosis: serology, ELISPOT for site-specific lymphocytes, urinary lipoarabinomannan, string test, and fine needle aspiration. Journal of Infectious Diseases, vol. 204, no. 4, pp. S1130-S1141.

[10] Purohit, M. and Mustafa, T. (2015). Laboratory diagnosis of extra-pulmonary tuberculosis (EPTB) in resource-constrained setting: state of the art, challenges and the need. Journal of Clinical and Diagnostic Research, vol. 9, no. 4, pp. EE01-EE06.

[11] Gupta, B. (2010). Sensitivity, specificity, negative and positive predictive values of adenosine deaminase in patients of tubercular and non-tubercular serosal effusion in India. Journal of Clinical Medicine Research, vol. 2, no. 3, pp. 121-126.

[12] Greco, S., Girardi, E., Masciangelo, R., et al. (2003). Adenosine deaminase and interferon gamma measurements for the diagnosis of tuberculous pleurisy: a metaanalysis. International Journal of Tuberculosis and Lung Disease, vol. 7, no. 8, pp. 777-786

[13] Dasgupta, A., Ghosh, R. N., Poddar, A. K., et al. (1994). Fine needle aspiration cytology of cervical lymphadenopathy with special reference to tuberculosis. Journal of Indian Medical Association, vol. 92, no. 2, pp. 44-46.

[14] Piras, M. A., Gakis, C., Budroni, M., et al. (1978). Adenosine deaminase activity in pleural effusions: an aid to differential diagnosis. British Medical Journal, vol. 2, no. 6154, pp. 1751-1752.

[15] Mugulkod, P., Chavan S., S. (2017). Serum adenosine deaminase levels and other laboratory parameters in the diagnosis of extrapulmonary tuberculosis: a clinicopathological study. International Journal of Research in Medical Sciences, vol. 5 , no. 7, p. 3140 .

[16] Khajuria, R., Goswami, K. C., Singh, K., et al. (2006). Pattern of lymphadenopathy on fine needle aspiration cytology in Jammu. JK Science, vol. 8, no. 3, pp. 157-159.

[17] Purohit, M. R., Mustafa, T., Mørkve, O., et al. (2009). Gender differences in the clinical diagnosis of tuberculous lymphadenitis-a hospital-based study from central India. International Journal of Infectious Diseases, vol. 13, no. 5, pp. 600-605. 
[18] Bhattacharya, S., Raghuveer, C. V., and Adhikari, P. (1998). FNAC diagnosis of tuberculosis-an eight years study at Mangalore. Indian Journal of Medical Sciences, vol. 52, no. 11, pp. 498-506.

[19] Khanna, A., Khanna, M., and Manjari, M. (2013). Cytomorphological patterns in the diagnosis of tuberculous lymphadenitis. International Journal of Medical and Dental Sciences, vol. 2, no. 2, pp. 182-188.

[20] Nassaji, M., Azarhoush, R., Ghorbani, R., et al. (2014). Acid fast staining in formalinfixed tissue specimen of patients with extrapulmonary tuberculosis. International Journal of Scientific and Research Publication, vol. 4, no. 1, pp. 2250-3153.

[21] Sulakshana, M. S., Ahmed, S. M., and Jayakumar, C. K. (2015). Study of serum adenosine deaminase levels in FNAC confirmed cases of tuberculous lymphadenitis. International Journal of Recent Advances in Multidisciplinary Research, vol. 2, no. 6, pp. 0476-0480.

[22] Ahmed, I., Sabah, S., Alam, B., et al. (2020). Role of serum ADA in patients of extra-pulmonary tuberculosis in a tertiary care hospital of Bangladesh. International Journal of Medical Research Professionals, vol. 6, no. 3, pp. 63-67.

[23] Alaarag, A., Mohammad, O., and Farag, N. (2016). Diagnostic utility of serum adenosine deaminase level in the diagnosis of pulmonary tuberculosis. Egyptian Journal of Bronchology, vol. 10, no. 2, pp. 133-139.

[24] Conde, M. B., Marinho, S. R., De Fatima Pereira, M., et al. (2002). The usefulness of serum adenosine deaminase 2 (ADA2) activity in adults for the diagnosis of pulmonary tuberculosis. Respiratory Medicine, vol. 96, no. 8, pp. 607-610.

[25] Salmanzadeh, S., Tavakkol, H., Bavieh, K., et al. (2015). Diagnostic value of serum adenosine deaminase (ADA) level for pulmonary tuberculosis. Jundishapur Journal of Microbiology, vol. 8, no. 3, p. e21760.

[26] Farazi, A., Moharamkhani, A., and Sofian, M. (2013). Validity of serum adenosine deaminase in diagnosis of tuberculosis. Pan African Medical Journal, vol. 15, p. 133. 